\title{
Optical properties of calcium under pressure from first-principles calculations
}

\author{
Ion Errea, ${ }^{1,2,3, *}$ Bruno Rousseau, ${ }^{2,3, \dagger}$ Asier Eiguren, ${ }^{1,2}$ and Aitor Bergara ${ }^{1,2,3}$ \\ ${ }^{1}$ Materia Kondentsatuaren Fisika Saila, Zientzia eta Teknologia Fakultatea, Euskal Herriko Unibertsitatea UPV/EHU, \\ 644 Postakutxatila, 48080 Bilbao, Basque Country, Spain \\ ${ }^{2}$ Donostia International Physics Center (DIPC), Manuel de Lardizabal Pasealekua 4, 20018 Donostia, Basque Country, Spain \\ ${ }^{3}$ Centro de Física de Materiales CFM-Materials Physics Center MPC, Centro Mixto CSIC-UPVIEHU, \\ Manuel de Lardizabal Pasealekua 5, 20018 Donostia, Basque Country, Spain
}

(Received 5 March 2012; revised manuscript received 29 June 2012; published 3 August 2012)

\begin{abstract}
Here we present theoretical ab initio calculations based on time-dependent density-functional theory of the macroscopic dielectric function of calcium from 0 to $110 \mathrm{GPa}$ in the fcc, bcc, and sc phases. All the dielectric functions are calculated using very fine reciprocal space meshes since both eigenvalues and matrix elements entering in the density-response functions are interpolated using Wannier functions. Our calculations correctly predict the energies of the low- and high-energy plasmons observed in the fcc phase at room pressure. Moreover, we predict that in the sc phase a very low-energy interband plasmon emerges at around $50 \mathrm{GPa}$ that dramatically modifies the behavior of the reflectivity making it almost vanish at the plasmon energy. As a consequence, calcium becomes an example of how optical properties can become complex under pressure.
\end{abstract}

DOI: 10.1103/PhysRevB.86.085106

PACS number(s): 31.15.A-, 71.45.Gm, 78.20.Bh, 62.50.-p

\section{INTRODUCTION}

Calcium is one of the most remarkable and interesting elements under pressure. Its behavior under compression represents a textbook example of how pressure can induce the emergence of complex and unexpected phenomena in elements that are a priori thought to be simple. ${ }^{1}$ Calcium undergoes a series of unsuspected phase transitions to lower coordinated structures. Room-temperature x-ray diffraction measurements ${ }^{2-5}$ demonstrate that the face-centered cubic (fcc) phase transforms to body-centered cubic (bcc) structure at $19 \mathrm{GPa}$. Subsequent phase transitions to simple cubic (sc), $P 4_{3} 2_{1} 2, C m c a$, and Pnma structures are found at 32, 119,143 , and $158 \mathrm{GPa}$, respectively. Although the sc phase shows imaginary phonon branches throughout the whole first Brillouin zone (1BZ) in ab initio calculations based on the harmonic approximation ${ }^{6-9}$ and alternative phases have been proposed, ${ }^{10,11}$ recent experiments reconfirm the presence of this phase in nature at room temperature. ${ }^{12,13}$ Also, novel theoretical calculations suggest that anharmonicity stabilizes the sc phase. ${ }^{14}$ Second, the electronic properties of calcium conflict with the naive idea that pressure makes metals better conductors. Despite being a good metal at ambient conditions, calcium falls to a semiconductor state in the fcc phase above $14 \mathrm{GPa}$ and recovers its metallic character upon transformation to the bcc phase. ${ }^{15}$ Finally, it is worth mentioning that calcium is already a superconducting material in the sc phase ${ }^{16}$ and becomes the element with the largest $T_{c}$ at higher pressures, reaching extraordinary values of $T_{c}$ such as $29 \mathrm{~K}$ at $216 \mathrm{GPa} .{ }^{17}$

Besides the mentioned structural phase transitions, the loss of metallic character, and the emergence of superconducting phenomena, the complexity induced by pressure in simple elements is also exemplified by the evolution of the optical properties under compression. As an example, the semiconductor character of materials, such as lithium or sodium at respective pressure ranges of $65-95 \mathrm{GPa}^{18,19}$ and $>200 \mathrm{GPa}^{20}$ is directly evidenced by the loss of shininess of the sample. Moreover, for the metallic oP8 and tl19 phases of $\mathrm{Na}^{21}$ a drastic modification of the high and uniform reflectivity associated to a good metal is observed. ${ }^{22}$ Theoretical calculations describe a similar mitigation of the reflectivity for fcc lithium, ${ }^{23}$ which is directly associated to the interband low-energy plasmon already predicted in previous $a b$ initio calculations. ${ }^{23-25}$ Indeed, in systems such as $\mathrm{Ag},{ }^{26,27}$ graphite, ${ }^{28}$ single-wall carbon nanotubes, ${ }^{29}$ or $\mathrm{MgB}_{2},{ }^{30,31}$ interband transitions in the band structure shape the dielectric function of metals and induce the emergence of several plasmons that cannot be understood within the free-electron model. If the origin of a plasmon resides in these interband transitions, it is sometimes referred to in the literature as an interband plasmon. ${ }^{32,33} \mathrm{We}$ will employ the expression "interband plasmon" with this in mind in what follows. Moreover, interband transitions are generally enhanced under pressure. This is generally due to the induced electronic localization reflected in the flattening of the bands. ${ }^{34,35}$ Therefore, the emergence of low-energy plasmons affecting the reflectivity coefficient might be a rather common phenomena under pressure. In this context, it is worth mentioning the recently reported ab initio calculations predicting a vanishing reflectivity in the infrared regime. ${ }^{36}$

Considering the mentioned outstanding properties of compressed calcium, it is sensible to ask whether its optical properties are as well characterized by the presence of low-energy plasmons leading to a complex and diminished reflectivity. In this work we present $a b$ initio calculations within time-dependent density-functional theory (TDDFT) for the dielectric and optical properties of $\mathrm{Ca}$ in a wide pressure range going from 0 to $110 \mathrm{GPa}$ and covering the fcc, bcc, and sc phases. In all calculations the dielectric function has been computed using very fine Brillouin zone integration k-point meshes taking advantage of the so-called Wannier interpolation. ${ }^{37,38}$ As will be shown, calcium is predicted to develop a low-energy plasmon under pressure dramatically modifying the optical properties and making the reflectivity almost vanish at around $0.9 \mathrm{eV}$ in the sc phase. According to our calculations the plasmon emerges around $50 \mathrm{GPa}$ with practically an infinite lifetime and appears completely damped above $95 \mathrm{GPa}$. Hence, our calculations demonstrate 
that calcium is another example of how pressure can induce the emergence of interband low-energy plasmons and emphasize that these can be rather common in many different compounds under compression.

The paper is organized as follows. In Sec. II we introduce the technical details of the $a b$ initio calculations of the dielectric and the optical properties using Wannier functions. The results of the calculations are presented and analyzed in Sec. III. Finally, summary and conclusions are given in Sec. IV. Unless otherwise specified atomic units are used throughout, namely, $\hbar=e^{2}=m_{e}=4 \pi \epsilon_{0}=1$.

\section{CALCULATION DETAILS}

Although the theorems that sustain density-functional theory (DFT) ${ }^{39}$ apply only to the ground state, TDDFT has been rigorously formulated to describe time-dependent systems and obtain the excitation spectrum of many-electron systems. ${ }^{40,41}$ In particular, considering their large screening capability and the consequent suppression of exchange-correlation effects, TDDFT has been successful in the calculation of plasmons in metals. ${ }^{42}$ Within this theory the density-response function is the central ingredient allowing for a detailed study of the possible collective excitations. The density-response function describes up to linear order the change in the electronic density induced by an external potential. In momentum space it can be calculated from the following Dyson-like matrix equation:

$$
\begin{aligned}
\chi_{\mathbf{G G}^{\prime}}(\mathbf{q}, \omega)= & \chi_{\mathbf{G G}^{\prime}}^{0}(\mathbf{q}, \omega)+\sum_{\mathbf{G}_{1} \mathbf{G}_{2}} \chi_{\mathbf{G G}_{\mathbf{1}}}^{0}(\mathbf{q}, \omega) \\
& \times\left[\frac{4 \pi}{\left|\mathbf{q}+\mathbf{G}_{1}\right|^{2}} \delta_{\mathbf{G}_{1} \mathbf{G}_{2}}+f_{\mathbf{G}_{1} \mathbf{G}_{2}}^{x c}(\mathbf{q}, \omega)\right] \chi_{\mathbf{G}_{2} \mathbf{G}^{\prime}}(\mathbf{q}, \omega) .
\end{aligned}
$$

In Eq. (1) $\chi_{\mathbf{G G}^{\prime}}^{0}(\mathbf{q}, \omega)$ represents the so-called noninteracting density-response function for a given momentum $\mathbf{q}$ and frequency $\omega$ of the external perturbation. The $\mathbf{G}$ vectors denote the reciprocal lattice vectors, and, $f_{\mathbf{G}_{1} \mathbf{G}_{2}}^{x c}(\mathbf{q}, \omega)$ encodes all the dynamical exchange-correlation effects. The noninteracting density-response function can be calculated from the singleparticle Kohn-Sham states as ${ }^{43}$

$$
\begin{aligned}
\chi_{\mathbf{G G}^{\prime}}^{0}(\mathbf{q}, \omega)= & \frac{1}{\Omega} \sum_{\mathbf{k}}^{1 B Z} \sum_{n m} \frac{f_{n \mathbf{k}}-f_{m \mathbf{k}+\mathbf{q}}}{\varepsilon_{n \mathbf{k}}-\varepsilon_{m \mathbf{k}+\mathbf{q}}+\omega+i \eta} \\
& \times\left\langle\phi_{n \mathbf{k}}\left|e^{-i(\mathbf{q}+\mathbf{G}) \cdot \mathbf{r}}\right| \phi_{m \mathbf{k}+\mathbf{q}}\right\rangle\left\langle\phi_{m \mathbf{k}+\mathbf{q}}\left|e^{i\left(\mathbf{q}+\mathbf{G}^{\prime}\right) \cdot \mathbf{r}}\right| \phi_{n \mathbf{k}}\right\rangle,
\end{aligned}
$$

where $\left|\phi_{n \mathbf{k}}\right\rangle$ and $\varepsilon_{n \mathbf{k}}$ are, respectively, the single-particle Kohn-Sham functions and energies, $f_{n \mathbf{k}}$ is the Fermi-Dirac distribution function, and $\eta$ is a positive infinitesimal that ensures causality. In this work, instead of computing $\chi^{0}$ directly through Eq. (2), what is explicitly computed is only the imaginary part given by

$$
\begin{aligned}
\operatorname{Im} \chi_{\mathbf{G G}^{\prime}}^{0}(\mathbf{q}, \omega)= & -\pi \frac{1}{\Omega} \sum_{\mathbf{k}}^{1 B Z} \sum_{n m}\left(f_{n \mathbf{k}}-f_{m \mathbf{k}+\mathbf{q}}\right) \\
& \times \delta\left(\varepsilon_{n \mathbf{k}}-\varepsilon_{m \mathbf{k}+\mathbf{q}}+\omega\right)\left\langle\phi_{n \mathbf{k}}\left|e^{-i(\mathbf{q}+\mathbf{G}) \cdot \mathbf{r}}\right| \phi_{m \mathbf{k}+\mathbf{q}}\right\rangle \\
& \times\left\langle\phi_{m \mathbf{k}+\mathbf{q}}\left|e^{i\left(\mathbf{q}+\mathbf{G}^{\prime}\right) \cdot \mathbf{r}}\right| \phi_{n \mathbf{k}}\right\rangle
\end{aligned}
$$

and the real part $\operatorname{Re} \chi_{\mathbf{G G}^{\prime}}^{0}(\mathbf{q}, \omega)$ is obtained by applying the Kramers-Kronig relations. ${ }^{44}$

Once $\chi^{0}$ has been computed following this procedure, the density-response function has been obtained through Eq. (1). The inverse dielectric matrix can be calculated from the density-response function as

$$
\epsilon_{\mathbf{G G}^{\prime}}^{-1}(\mathbf{q}, \omega)=\delta_{\mathbf{G G}^{\prime}}+\frac{4 \pi}{|\mathbf{q}+\mathbf{G}|^{2}} \chi_{\mathbf{G G}^{\prime}}(\mathbf{q}, \omega) .
$$

The quantity $-\operatorname{Im} \epsilon_{\mathbf{G G}}^{-1}(\mathbf{q}, \omega)$ is usually known as the energyloss function, and the collective excitations of a system can in principle be identified from its peaks. If a plasmon is defined as a self-sustained charge oscillation, that is, as an oscillating electronic potential that is set up without the application of an external potential, plasmonic resonances appear as peaks in the energy-loss function. ${ }^{45}$ In simple metals there is one single plasmon resonant peak, whose frequency is completely determined by the density of the valence electrons, but in more complex metals band structure effects complicate the profile of the energy-loss function, and several plasmon peaks may appear. The optical properties of solids are related to the vanishing momenta limit of the dielectric function. This limit is often referred as the macroscopic dielectric function and is defined as

$$
\epsilon^{M}(\omega)=\lim _{\mathbf{q} \rightarrow 0} \frac{1}{\epsilon_{00}^{-1}(\mathbf{q}, \omega)},
$$

where $\epsilon_{00}^{-1}(\mathbf{q}, \omega)$ is the inverse dielectric matrix element with $\mathbf{G}=0$ and $\mathbf{G}^{\prime}=0$.

In case that crystal local-field effects (CLFEs) are neglected, one obtains that $\epsilon^{M}(\omega)=\lim _{\mathbf{q} \rightarrow 0} \epsilon(\mathbf{q}, \omega)$, but, according to the definition of Eq. (5), CLFEs are included in $\epsilon^{M}(\omega)$ through the inversion of the dielectric matrix. The refraction index $n$ and the extinction coefficient $\kappa$ are related to the macroscopic dielectric function through the equations

$$
\begin{aligned}
& \operatorname{Re} \epsilon^{M}(\omega)=n^{2}(\omega)-\kappa^{2}(\omega), \\
& \operatorname{Im} \epsilon^{M}(\omega)=2 n(\omega) \kappa(\omega) .
\end{aligned}
$$

Once these coefficients are known, the reflectivity $R$ is defined by

$$
R(\omega)=\frac{(n(\omega)-1)^{2}+\kappa^{2}(\omega)}{(n(\omega)+1)^{2}+\kappa^{2}(\omega)} .
$$

The convergence of the sum over the $\mathbf{k}$ points in Eq. (3) represents generally a very difficult task, and a very large number of $\mathbf{k}$ points might be needed. Correspondingly, the socalled Wannier interpolation method can be a very interesting way to overcome this problem, since it allows for a very accurate and efficient sampling of the reciprocal space. ${ }^{46,47}$ The first step in the calculation is to obtain a reasonable Kohn-Sham effective potential through a standard DFT self-consistent calculation. In a second step, the Kohn-Sham states are calculated non-self-consistently on a relatively coarse mesh from which the Wannier interpolation is accessible. In this work we considered the QUANTUM-ESPRESSO package ${ }^{48}$ for both the self-consistent and non-self-consistent calculations. The obtained Kohn-Sham energies are interpolated into a very fine k-point mesh through the Wannier interpolation. 
The WANNIER90 $\operatorname{code}^{49}$ has been used for this purpose. The eigenenergies are not the only ingredients needed in Eq. (3); the matrix elements also need to be interpolated. The matrix elements, $\rho_{n m}(\mathbf{k}, \mathbf{q}, \mathbf{G}) \equiv\left\langle\phi_{n \mathbf{k}}\left|e^{-i(\mathbf{q}+\mathbf{G}) \cdot \mathbf{r}}\right| \phi_{m \mathbf{k}+\mathbf{q}}\right\rangle$, are first calculated for the $\mathbf{k}$ points of the coarse mesh. These matrix elements are then "Wannier rotated" as

$$
\rho_{n^{\prime} m^{\prime}}^{W}(\mathbf{k}, \mathbf{q}, \mathbf{G})=\sum_{n m}^{N_{W}} U_{n^{\prime} n}^{*}(\mathbf{k}) \rho_{n m}(\mathbf{k}, \mathbf{q}, \mathbf{G}) U_{m m^{\prime}}(\mathbf{k}+\mathbf{q}),
$$

where the $U$ are the Wannier unitary matrices and $N_{W}$ the number of bands chosen to build the maximally localized Wannier functions. The aim is to obtain the $\rho_{n m}(\mathbf{k}, \mathbf{q}, \mathbf{G})$ matrix elements for fixed $n m, \mathbf{q}$, and $\mathbf{G}$ indexes in a fine $\mathbf{k}$-point mesh. The $\rho_{n m}(\mathbf{k}, \mathbf{q}, \mathbf{G})$ matrix elements are not smooth due to the "random phase" carried by the Kohn-Sham states. On the contrary, Wannier rotated $\rho_{n^{\prime} m^{\prime}}^{W}(\mathbf{k}, \mathbf{q}, \mathbf{G})$ matrix elements show a smooth dependence on $\mathbf{k}$ and can be efficiently interpolated into a very fine mesh, considering, for example, a trilinear interpolation algorithm. After performing the interpolation, if $\mathbf{k}^{\prime}$ is a point of the fine mesh, the matrix element in the Kohn-Sham basis at $\mathbf{k}^{\prime}$ can be obtained simply as

$$
\rho_{n m}\left(\mathbf{k}^{\prime}, \mathbf{q}, \mathbf{G}\right)=\sum_{n^{\prime} m^{\prime}}^{N_{W}} U_{n n^{\prime}}\left(\mathbf{k}^{\prime}\right) \rho_{n^{\prime} m^{\prime}}^{W}\left(\mathbf{k}^{\prime}, \mathbf{q}, \mathbf{G}\right) U_{m^{\prime} m}^{*}\left(\mathbf{k}^{\prime}+\mathbf{q}\right) .
$$

The $U\left(\mathbf{k}^{\prime}\right)$ and $U\left(\mathbf{k}^{\prime}+\mathbf{q}\right)$ matrices in Eq. (10) are directly obtained in the Wannier-interpolation step as they represent the electron wave functions in the basis of the Wannier functions.

Another advantage of Wannier functions is that they can be used to design an adaptive smearing scheme. ${ }^{47}$ Indeed, in our calculations the Dirac delta distribution in Eq. (3) has been approximated by a Gaussian function $\left(\sqrt{\pi \sigma_{n m, \mathbf{k}}^{2}}\right)^{-1} \exp \left(-x^{2} / \sigma_{n m, \mathbf{k}}^{2}\right)$, where $\sigma_{n m, \mathbf{k}}$ is a statedependent smearing parameter adapted to the steepness of the integrand formed by $\varepsilon_{n \mathbf{k}}-\varepsilon_{m \mathbf{k}+\mathbf{q}}+\omega$. The optimal values of $\sigma_{n m, \mathbf{k}}$ are easily deduced from the band derivatives, which are readily available in the Wannier interpolation method. ${ }^{46}$ Therefore, within this procedure the smearing parameter is not an external input parameter anymore, but a parameter adapted automatically to resolve sharp features in the spectral function.

In all our calculations CLFEs have been treated including four reciprocal lattice shells in the calculation of the density-response function for both fcc and sc structures. We considered three shells for the bcc structure. We have made use of the random-phase approximation (RPA), namely, $f^{x c}=0$, in all calculations. The Kohn-Sham states entering Eq. (3) have been calculated using a plane-wave basis with a 100 Ry energy cutoff and making use of the generalized gradient approximation (GGA) for the exchange-correlation potential. ${ }^{50}$ The electron-ion interaction has been modeled with a norm-conserving pseudopotential including $3 s^{2}, 3 p^{6}$, and $4 s^{2}$ states in the valence. The integrations over the $1 \mathrm{BZ}$ needed to converge the effective Kohn-Sham potentials have been performed using a $16 \times 16 \times 16$ Monkhorst-Pack mesh in all cases. The coarse meshes used for the interpolation are $20 \times 20 \times 20,16 \times 16 \times 16$, and $24 \times 24 \times 24$ for the fcc, bcc, and sc structures, respectively. A $144 \times 144 \times 144$ fine mesh has been chosen for the Wannier interpolation in all cases. In the sum over band indexes in Eq. (3) all bands up to $30 \mathrm{eV}$ above the Fermi energy were included. The $\mathbf{q} \rightarrow 0$ limit in Eq. (5) was treated in all cases by simply considering a finite but sufficiently small value of $|\mathbf{q}|$ ranging from 0.01 to 0.02 a.u. $^{-1}$.

\section{RESULTS AND DISCUSSION}

Following the procedure outlined in Sec. II here we will present and discuss the results that we have obtained for the optical properties of calcium in the fcc phase at ambient pressure, in the bcc phase at $20 \mathrm{GPa}$, and in the sc phase at $35,50,65,80,95$, and $110 \mathrm{GPa}$. Hence, we will cover all the stability range of these three phases.

\section{A. The fcc phase}

As shown in Fig. 1, the energy-loss spectra of fcc $\mathrm{Ca}$ at ambient pressure is not at all as trivial as in a free-electronlike metal. Indeed, the presence of two clear bulk plasmons instead of the single one expected within the free-electron-like approach is remarkable. The first plasmon peak appears at an energy of $3.7 \mathrm{eV}$, while the second emerges at approximately $8.3 \mathrm{eV}$. When CLFEs are neglected, the energy of the lowenergy plasmon is shifted less than $1 \%$, while the energy of the second around $3 \%$. We deduce that CLFEs are not too relevant for determining the plasmon energy at least for small momenta. Considering that in the free-electron model the energy of the intraband plasmon is given as

$$
\omega_{p}=\sqrt{4 \pi n},
$$

where $n$ is the valence electron density, the intraband plasmon is expected around $\omega_{p}=8.0 \mathrm{eV}$. Hence, while the plasmon at $8.3 \mathrm{eV}$ seems to be associated to intraband transitions, the lower energy plasmon must be associated to interband transitions not present in the free-electron model. Let us denote henceforth this plasmon as the interband plasmon. Remarkably, different measurements find a plasmon peak

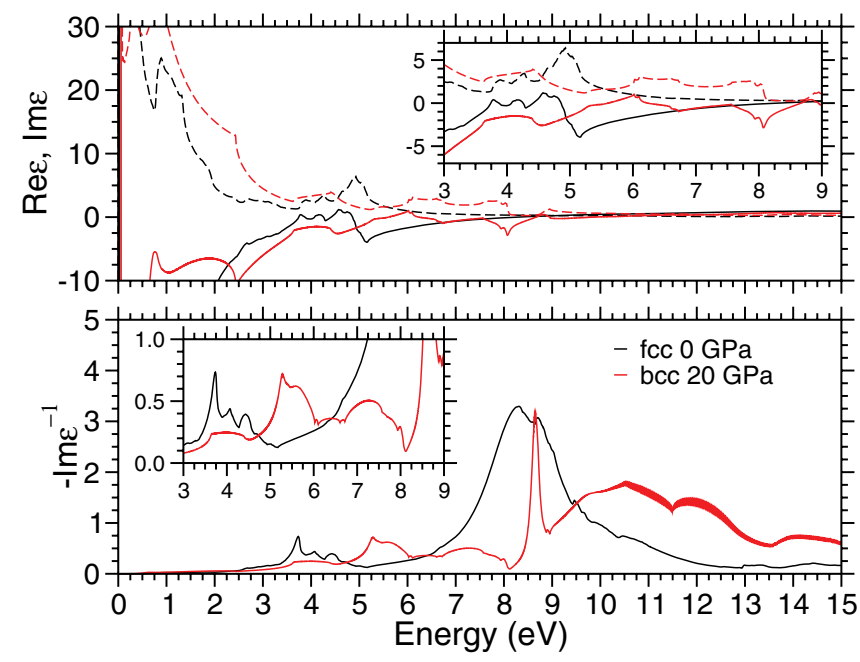

FIG. 1. (Color online) In the top panel $\operatorname{Re} \epsilon^{M}(\omega)$ (solid line) and $\operatorname{Im} \epsilon^{M}(\omega)$ (dashed line) are plotted for the fcc phase of $\mathrm{Ca}$ at $0 \mathrm{GPa}$ and for the bcc phase of $\mathrm{Ca}$ at $20 \mathrm{GPa}$. In the bottom panel the energy-loss function is shown at these pressures for these two structures. In both figures the inset addresses the 3-9 eV energy range. 


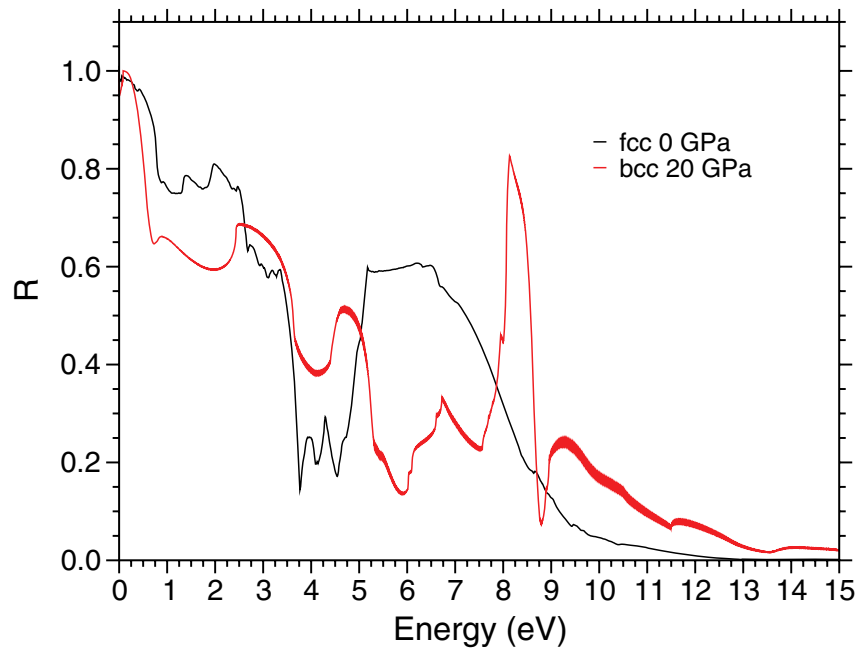

FIG. 2. (Color online) The reflectivity of fcc $\mathrm{Ca}$ at $0 \mathrm{GPa}$ and of bcc $\mathrm{Ca}$ at $20 \mathrm{GPa}$.

between 3.4 and $4.4 \mathrm{eV}$, and a second one between 8.8 and $9.8 \mathrm{eV} .^{51-54}$ These values are in reasonable agreement with our calculations.

The reflectivity of fcc $\mathrm{Ca}$ at ambient pressure is plotted in Fig. 2. At low energies the reflectivity is slightly suppressed in a series of steps. Each step coincides with the gaps for interband transitions at high-symmetry points $\mathrm{X}$ and $\mathrm{L}$. At these points the joint density of states is large, and interband transitions suppress the reflectivity as suggested by Ehrenreich and Philipp. ${ }^{26,55}$ Although up to approximately $3.5 \mathrm{eV}$ the reflectivity is rather strong, close to the interband plasmon energy the reflectivity coefficient shows a sudden dip, indicating that plasma oscillations induce its most remarkable features. At higher energies the reflectivity rises, but it is suppressed once again when it reaches the energy of the intraband plasmon. A similar behavior of the reflectivity has been found experimentally. ${ }^{51}$ The agreement found for the plasmon energies and the profile of the reflectivity suggests that our method based on Wannier interpolation can accurately describe the optical properties of $\mathrm{Ca}$ at higher pressures, in different phases where no experiments are available as far as the authors know.

The energy of the interband plasmon coincides with the energy at which $\operatorname{Re} \epsilon^{M}(\omega)$ passes through zero while $\operatorname{Im} \epsilon^{M}(\omega)$ remains weak. The optical interband electronic transitions of about $5 \mathrm{eV}$ between occupied and unoccupied states along XWLK generates a peak in $\operatorname{Im} \epsilon^{M}(\omega)$ around this energy. As it can be appreciated in the band structure plotted in Fig. 3(a), these transitions happen between rather localized bands. Driven by the Kramers-Kronig relations, this increase in $\operatorname{Im} \epsilon^{M}(\omega)$ makes $\operatorname{Re} \epsilon^{M}(\omega)$ pass through zero at the plasmon frequency and induces the sudden dip of the reflectivity mentioned above. Indeed, the role of interband transitions in the emergence of a plasmon, which is accompanied with the abrupt modification of the reflectivity, is framed in the context described by Ehrenreich and Philipp. ${ }^{26,55}$

The presence of the above-mentioned peak in the imaginary part of the macroscopic dielectric function is related to an unconventional behavior of the valence electrons. Contrary to what is expected for a free-electron-like metal, here valence
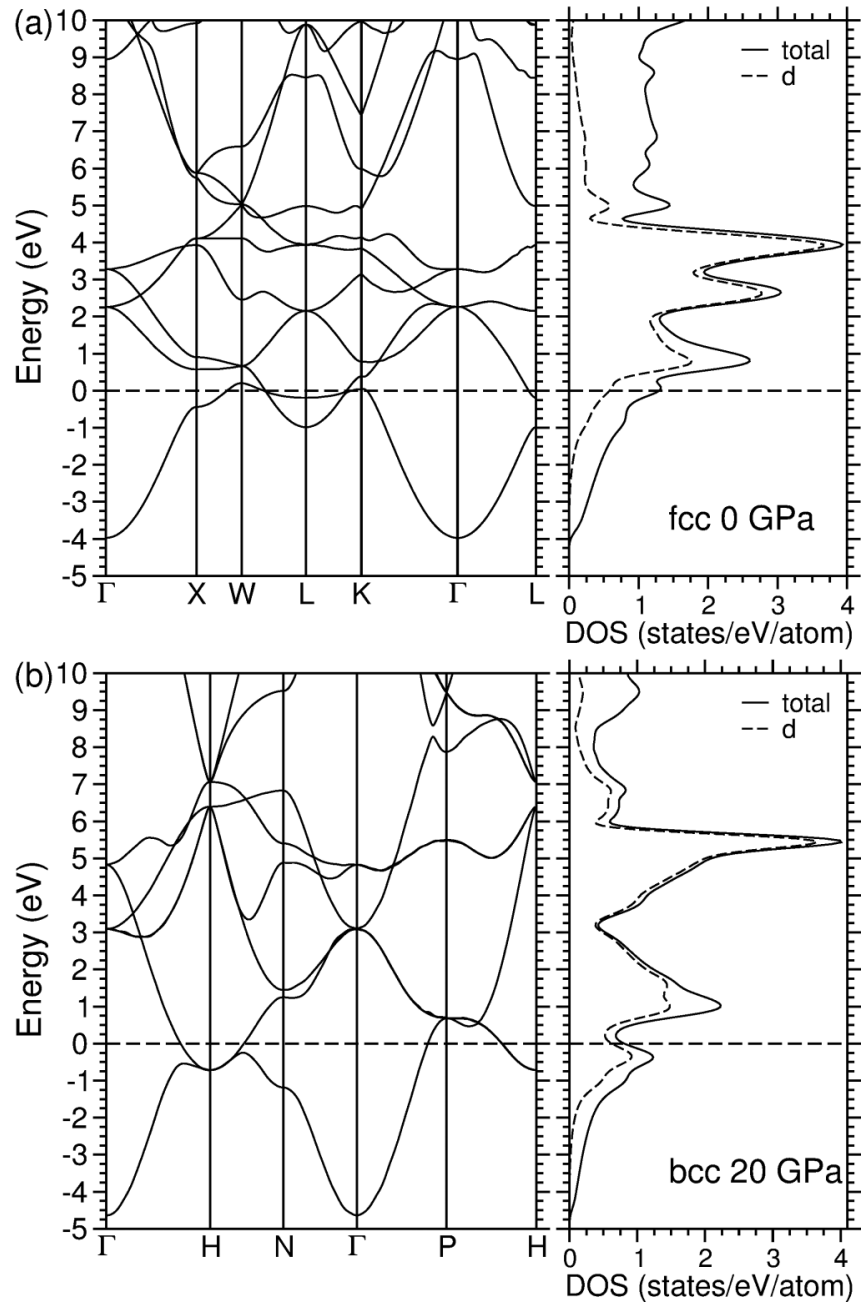

FIG. 3. (a) Band structure of fcc $\mathrm{Ca}$ at $0 \mathrm{GPa}$. The total DOS and its projection onto $d$ states are shown in the right panel. (b) Band structure and DOS of bcc Ca at $20 \mathrm{GPa}$. The Fermi level is depicted with a dashed line in all figures.

electrons localize in the interstitial octahedral sites, already at room pressure in fcc calcium. This fact is reflected by the electron localization function (ELF), which is a simple and useful tool to measure the electronic degree of localization. ${ }^{56}$ Figure 4(a) demonstrates that the ELF has, in fact, a local maximum at the octahedral site. As a consequence, the fcc $\mathrm{Ca}$ bands show a rather flat structure leading to the prominence of electronic transitions close to $5 \mathrm{eV}$. Let us remark as well that, as shown by the density of states (DOS) of Fig. 3(a), the $3 d$ states lie very close to the Fermi energy, and along the WLK high-symmetry line they appear even occupied. On the other hand, those occupied states lying close to the Fermi level along the XW high-symmetry line, have already a $d$ character, anticipating the $s \rightarrow d$ electronic transfer induced by pressure. Interestingly, when the fcc phase is compressed, the localization at the octahedral sites is increased, and a gap is opened at the Fermi level leading to a semiconductor state. ${ }^{10,15}$

\section{B. The bcc phase}

The metallic behavior is recovered when calcium transforms at $19 \mathrm{GPa}$ to the bcc phase. At this pressure the $s \rightarrow d$ 


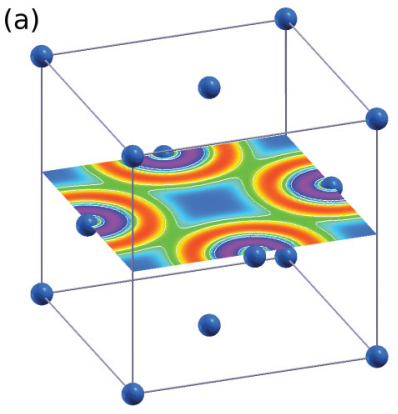

fCc $0 \mathrm{GPa}$

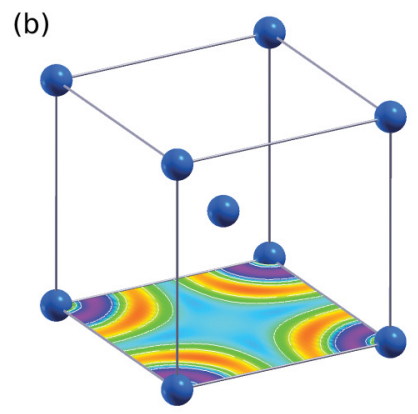

bcc $20 \mathrm{GPa}$

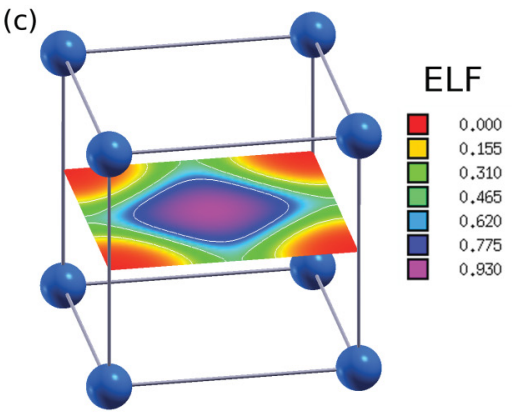

sc $35 \mathrm{GPa}$

FIG. 4. (Color online) ELF of the fcc phase of Ca at $0 \mathrm{GPa}$ (a), of the bcc phase at $20 \mathrm{GPa}$ (b), and of the sc phase at $35 \mathrm{GPa}$ (c). In (a) the octahedral sites are located at the center and at the corners of the plotted plane, while in (b) the octahedral site is located at the center of the plotted plane.

electronic transition is already complete. This can be seen in the DOS depicted in Fig. 3(b). The bcc band structure shows that the electrons are less localized than in the fcc phase. As noted in Fig. 4(b), this is also reflected in the lower value that the ELF takes in the octahedral sites for the bcc phase in comparison to the fcc phase. It seems that the localization is reduced in the less compact bcc structure since the valence electrons have more space. As a consequence, the electronic bands are slightly more dispersive and the peak observed in $\operatorname{Im} \epsilon^{M}(\omega)$ around $5 \mathrm{eV}$ disappears, describing instead a more homogeneous profile. Nevertheless, the profile of $\operatorname{Im} \epsilon^{M}(\omega)$ induces $\operatorname{Re} \epsilon^{M}(\omega)$ to go through zero, which results in a wide resonant peak in the energy-loss function close to $5.3 \mathrm{eV}$. The resonant peak is almost insensitive to CLFEs. For higher energies, the intraband-like plasmon is present but with a double-peak structure: a sharp feature at $8.7 \mathrm{eV}$ and a broad peak at $10.5 \mathrm{eV}$. In both cases the error made when neglecting CLFEs is less than $1 \%$. Although the $8.7 \mathrm{eV}$ peak is weaker than the ideal $\omega_{p}=10.5 \mathrm{eV}$ result derived using the valence electron density in Eq. (11), the broad peak matches the free-electron-like value.

The calculated energy-loss spectrum induces a reduction of the reflectivity coefficient rather continuously up to $8 \mathrm{eV}$, as noted in Fig. 2. Here the most important contribution comes from interband transitions. Contrary to fcc $\mathrm{Ca}$ at ambient conditions, in this case the onset of interband transitions is not associated to the contributions from high-symmetry points areas characterized by large joint DOS. As a consequence, the characteristic step-function-like behavior is smoothed out. As expected, the weakest value of the reflectivity is found close to the energies corresponding to the broad low-energy excitation. Then, the reflectivity coefficient describes a sharp increase until complete suppression after the high-energy plasmon frequencies are reached, exactly as shown for the fcc phase.

\section{The sc phase}

When Ca adopts a sc structure beyond $32 \mathrm{GPa}$, its energyloss spectra starts to exhibit several remarkable features. Indeed, as shown in Fig. 5, a very low-energy undamped plasmon emerges at approximately $1 \mathrm{eV}$. This plasmon may be similar to the one found in the high-pressure phases of $\mathrm{Na}^{22}$ as it appears in the same energy range. This remarkable plasmon shows an interesting evolution with pressure. At 35 GPa the plasmon still has not emerged, only a wide small peak is appreciated at $\sim 0.5 \mathrm{eV}$, but at $50 \mathrm{GPa}$ a clear peak is observed around $0.9 \mathrm{eV}$. One could say that at this pressure the plasmon is undamped considering that both imaginary and real parts of
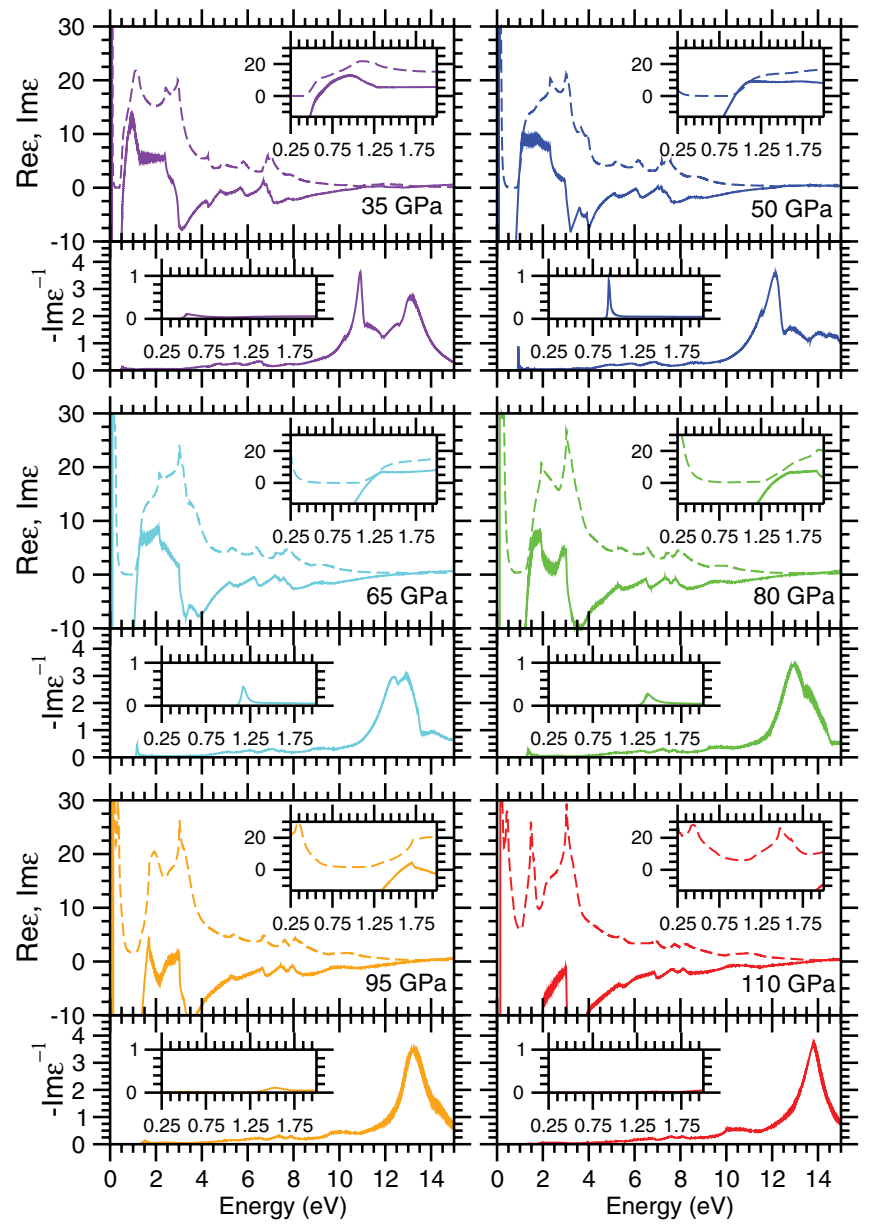

FIG. 5. (Color online) In the top panels $\operatorname{Re} \epsilon^{M}(\omega)$ (solid lines) and $\operatorname{Im} \epsilon^{M}(\omega)$ (dashed lines) are plotted for the sc phase of $\mathrm{Ca}$ at 35, $50,65,80,95$, and $110 \mathrm{GPa}$. In the bottom panels the energy-loss function is shown. In all figures, the inset addresses the $0.25-2.0 \mathrm{eV}$ energy range. 
$\epsilon^{M}(\omega)$ vanish practically at the plasmon energy, and that the condition for having a peak with vanishing line width (in case the CLFEs are neglected) is also fulfilled. ${ }^{45}$ This peak shifts to approximately $1.2 \mathrm{eV}$ at $65 \mathrm{GPa}$ and to $1.4 \mathrm{eV}$ at $80 \mathrm{GPa}$. As pressure is increased, the low-energy plasmon acquires a larger linewidth since, as depicted in Fig. 5, $\operatorname{Re} \epsilon^{M}(\omega)$ and $\operatorname{Im} \epsilon^{M}(\omega)$ no longer vanish at the same energy. Following this trend, at 95 and $110 \mathrm{GPa}$ the plasmon has already disappeared as no peak is observed in the energy-loss function. The inclusion of CLFEs does not significantly modify the plasmon energy position, similar to the interband plasmon found in fcc $\mathrm{Li}$ at small momenta. ${ }^{25}$ Besides the remarkable low-energy plasmon, higher energy plasmons are also present in the sc phase of $\mathrm{Ca}$. Although at $35 \mathrm{GPa}$ we find two prominent peaks above $10 \mathrm{eV}$, one at $10.9 \mathrm{eV}$ and the second at 13.1 $\mathrm{eV}$, at the other pressures the second peak is not observed, and only a regular intraband plasmon is present. Its energy is increased under compression in agreement with the density enhancement. Indeed, the simple model in Eq. (11) predicts the energy of this plasmon being not so far from the $a b$ initio results. Although CLFEs are practically irrelevant for determining the energy of the interband low-energy plasmon, their effect is larger for the high-energy plasmon. According to our results, neglecting CLFEs leads to an error of up to $6 \%$ in the intraband plasmon energy.

The effect in the reflectivity of the plasmons that we have characterized is illustrated in Fig. 6. At $35 \mathrm{GPa}$, although there is no plasmon in the energy-loss spectra, the reflectivity is suppressed around $0.5 \mathrm{eV}$, coinciding with the onset of interband transitions close to the high-symmetry $\mathrm{R}$ point (see Fig. 7 for band structure). Nevertheless, the presence of the low-energy plasmon induces a much more pronounced dip in $R$ at pressures that show a low-energy plasmon resonance. The smaller the line width of the plasmon, the more pronounced becomes the dip. For instance, at $50 \mathrm{GPa}$ the reflectivity almost vanishes at $0.9 \mathrm{eV}$. There is no such dramatic change in the reflectivity at low energies above $95 \mathrm{GPa}$, where the low-energy plasmon is no longer present and interband transitions define its profile. On the other hand, in all the stability range of the sc phase, the reflectivity is suppressed above the energy of the intraband plasmon.

As noted above, a peak in the energy-loss function is generally a consequence of the fact that $\operatorname{Re} \epsilon^{M}(\omega)=0$ when $\operatorname{Im} \epsilon^{M}(\omega)$ describes a valley where almost no electronic transitions are accessible, and it almost vanishes. This behavior is clearly characterized in the insets of Fig. 5 and is specially clear at $50 \mathrm{GPa}$, pressure at which the plasmon is practically undamped. Therefore, in order to understand the origin of the low-energy plasmon it is crucial to study which transitions contribute to the large increase of $\operatorname{Im} \epsilon^{M}(\omega)$ after the valley. Indeed, as suggested by the Kramers-Kronig relations, it is this region with large $\operatorname{Im} \epsilon^{M}(\omega)$ that is forcing $\operatorname{Re} \epsilon^{M}(\omega)$ to vanish at some point in the valley. ${ }^{26,55}$

In Fig. 7 the band structure of $\mathrm{sc} \mathrm{Ca}$ is shown for various pressures in its stability range. The calculated DOS demonstrates that most of the electron states acquire a $d$ character already at $35 \mathrm{GPa}$. The general features of the calculated DOS and its trend under pressure are in agreement with previous calculations. ${ }^{57}$ In order to identify which are the relevant low-energy optical transitions contributing to

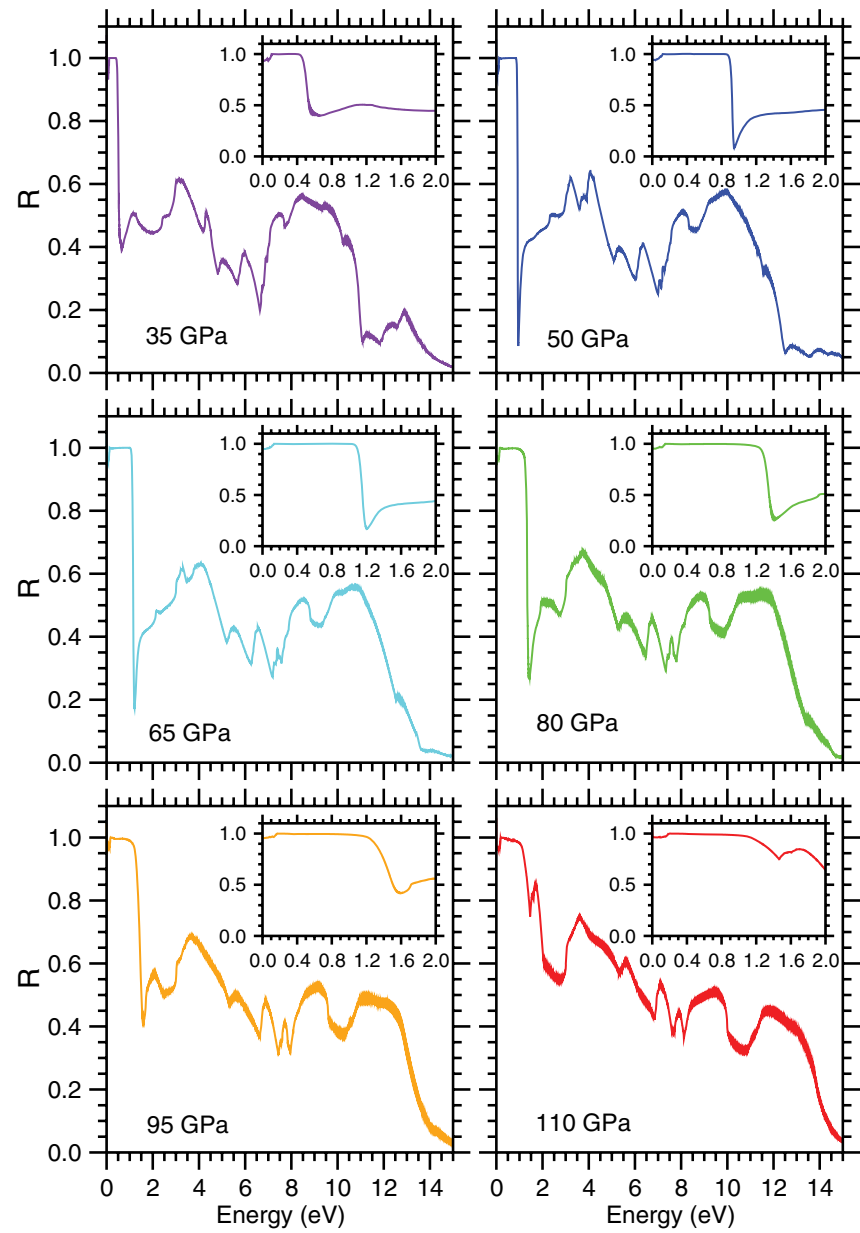

FIG. 6. (Color online) The reflectivity of $\mathrm{sc} \mathrm{Ca}$ at 35, 50, 65, 80, 95, and $110 \mathrm{GPa}$. The inset addresses the $0.0-2.0 \mathrm{eV}$ energy range. The slight deviation of the reflectivity from unity as $\omega \rightarrow 0$ is a numerical artifact caused by the use of a very small $\mathbf{q}$ vector to approximate the optical limit (see the end of Sec. II).

$\operatorname{Im} \epsilon^{M}(\omega)$ after the valley, we focus on the possible optical transitions below approximately $2 \mathrm{eV}$. Optical transitions with such a low energy happen in different regions of the 1BZ: close to $\Gamma$, along $\Gamma \mathrm{X}$ close to $\mathrm{X}$, along $\mathrm{XM}$ in the neighborhood of the band crossing, close to R, and halfway between $\Gamma \mathrm{M}$. At $35 \mathrm{GPa}$, the pocket at $\Gamma$ remains unoccupied, and, thus, no transitions are possible in this region. As pressure is increased, the pocket starts to be occupied, thus allowing optical transitions. The range of energies for these transitions is larger for higher pressures, but the starting energy for them is larger the greater the pressure. On the other hand, those transitions close to $\mathrm{X}$ along $\Gamma \mathrm{X}$ start at lower energies the higher the pressure. Regarding the transitions along XM, these are activated at lower energies for lower pressures, similar to the optical transitions close to the $\mathrm{R}$ point. These transitions, depicted with small arrows in Fig. 7, are greatly favored by the flat region found in the band structure along the high-symmetry line $\mathrm{R} \Gamma$ especially in the $35-80 \mathrm{GPa}$ pressure range. This flat region is reflected by the strong localization of the valence electrons in the interstitial regions as noted by the ELF plot in Fig. 4(c). Finally, the electronic transitions halfway between $\Gamma \mathrm{M}$ are relevant only for 95 and $110 \mathrm{GPa}$. 

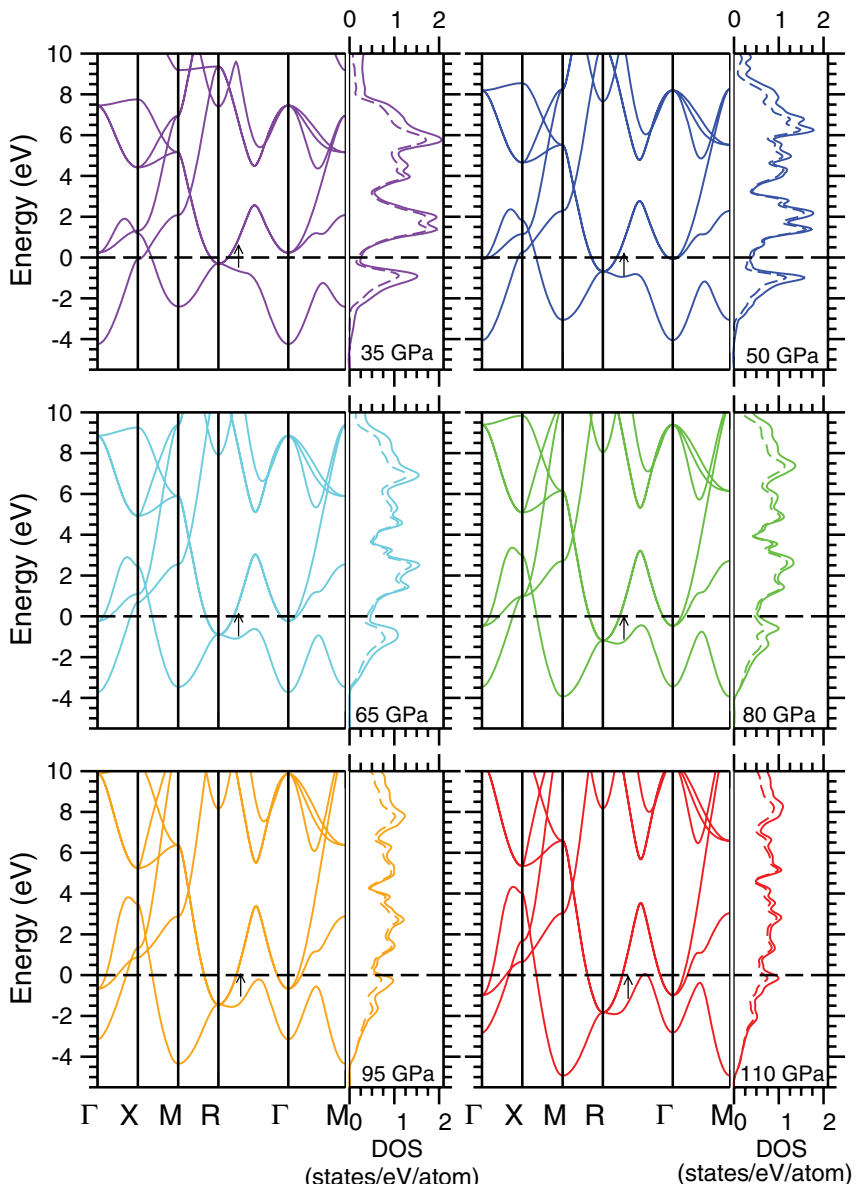

FIG. 7. (Color online) Band structure and DOS of sc Ca at 35, $50,65,80,95$, and $110 \mathrm{GPa}$. The projection of the DOS onto $d$ states is depicted with dashed lines. The Fermi level is depicted with a horizontal black dashed line. Interband transitions along $\mathrm{R} \Gamma$ are depicted with small arrows.

Interestingly, the increase of $\operatorname{Im} \epsilon^{M}(\omega)$ after the valley coincides exactly with the lowest energy for the optical transitions close to high-symmetry $\mathrm{R}$ point or along the high-symmetry line XM in the neighborhood of the band crossing. These transitions shift to higher energies under pressure, forcing the real part of the macroscopic dielectric function to have a zero at higher energies. On the other hand, it seems that the possible transitions close to $\Gamma$ are not too relevant for $\operatorname{Im} \epsilon^{M}(\omega)$. Indeed, if these transitions had a strong contribution to the imaginary part of the macroscopic dielectric function, the valley would not be present and the low-energy plasmon would also disappear. The reason why some transitions are reflected in the imaginary part and some others are apparently hidden might be related to the structure of the DOS. Indeed, while the flattened regions in the band structure both along $\mathrm{R} \Gamma$, associated to the strong localization in the interstitial sites, and $\Gamma \mathrm{M}$ seem to be reflected in the DOS, the states close to $\Gamma$ do not seem to contribute much to the DOS. Therefore, the evolution with pressure of the remarkable low-energy plasmon can be understood in terms of the evolution of the band structure under compression.

\section{CONCLUSIONS}

We have presented a theoretical $a b$ initio study of the optical properties of $\mathrm{Ca}$ under pressure. In all our calculation we have used Wannier interpolation in order to resolve the sharp features present in the spectral functions. The energies of the low- and high-energy plasmons that we predict for the fcc phase at room pressure are in good agreement with experiments. The most relevant features in our calculated reflectivity are also identified in measurements. ${ }^{51-54}$ The presence of the low-energy interband plasmon in this phase, which is related to the large localization of the valence electrons in the interstitial octahedral sites, addresses that fcc $\mathrm{Ca}$ is not a simple free-electron-like metal at room pressure. We predict that a low-energy plasmon remains in the bcc phase at $20 \mathrm{GPa}$, though at higher energies and with a larger line width. Finally, according to our calculations, the sc phase develops a remarkable very low-energy interband plasmon above $50 \mathrm{GPa}$. This induces an almost vanishing reflectivity in the infrared regime. The presence of this low-energy plasmon can be attributed to the electron state localization induced by pressure in the interstitial sites. This plasmon is almost undamped at this pressure, but acquires a larger line width under compression, and it is suppressed above $95 \mathrm{GPa}$. Plasmon dispersions and the reflectivity coefficients of materials are accessible today with great accuracy under pressure $;^{22,58,59}$ thus, it would be very interesting if experiments could confirm the presence of the low-energy plasmon predicted in this work for the sc phase.

In conclusion, this works strengthens the idea that pressureinduced electronic localization induces the appearance of low-energy plasmons in the energy-loss spectra. The presence of this type of plasmons strongly modifies the reflectivity, leading it to an almost vanishing value close to the plasmon energy. This anomalous optical behavior has been already observed in $\mathrm{Na}^{22}$ and has been predicted in $\mathrm{Li}^{23-25}$ and $\mathrm{AlH}_{3} .{ }^{36}$ Thus, calcium seems to be another example of this behavior, providing a good example of how pressure-induced complexity is also reflected in the optical properties.

\section{ACKNOWLEDGMENTS}

The authors are grateful to the Department of Education, Universities and Research of the Basque Government, UPV/EHU (Grant No. IT-366-07) and the Spanish Ministry of Science and Innovation (Grant No. FIS2010-19609-C02-00) for financial support. Computer facilities were provided by the DIPC.
*Present address: Institut de Minéralogie et de Physique des Milieux Condensé (IMPMC), Université Pierre et Marie Curie, case 115, 4 place Jussieu, 75252 Paris cedex 05, France.

†Present address: Université de Montréal, C.P. 6128, Montréal, Québec, Canada H3C 317.
${ }^{1}$ B. Rousseau, Y. Xie, Y. Ma, and A. Bergara, Eur. Phys. J. B 81, 1 (2011).

${ }^{2}$ H. Olijnyk and W. B. Holzapfel, Phys. Lett. A 100, 191 (1984).

${ }^{3}$ T. Yabuuchi, Y. Nakamoto, K. Shimizu, and T. Kikegawa, J. Phys. Soc. Jpn. 74, 2391 (2005). 
${ }^{4}$ H. Fujihisa, Y. Nakamoto, K. Shimizu, T. Yabuuchi, and Y. Gotoh, Phys. Rev. Lett. 101, 095503 (2008).

${ }^{5}$ Y. Nakamoto, M. Sakata, K. Shimizu, H. Fujihisa, T. Matsuoka, Y. Ohishi, and T. Kikegawa, Phys. Rev. B 81, 140106 (2010).

${ }^{6}$ I. Errea, M. Martinez-Canales, A. R. Oganov, and A. Bergara, High Press. Res. 28, 443 (2008).

${ }^{7}$ G. Gao, Y. Xie, T. Cui, Y. Ma, L. Zhang, and G. Zou, Solid State Commun. 146, 181 (2008).

${ }^{8}$ A. M. Teweldeberhan and S. A. Bonev, Phys. Rev. B 78, 140101 (2008).

${ }^{9}$ A. M. Teweldeberhan, J. L. Dubois, and S. A. Bonev, Phys. Rev. Lett. 105, 235503 (2010).

${ }^{10}$ A. R. Oganov, Y. Ma, Y. Xu, I. Errea, A. Bergara, and A. O. Lyakhov, Proc. Natl. Acad. Sci. USA 107, 7646 (2010).

${ }^{11}$ Y. Yao, D. D. Klug, J. Sun, and R. Martoňák, Phys. Rev. Lett. 103, 055503 (2009).

${ }^{12}$ Q. F. Gu, G. Krauss, Y. Grin, and W. Steurer, Phys. Rev. B 79, 134121 (2009).

${ }^{13}$ W. L. Mao, L. Wang, Y. Ding, W. Yang, W. Liu, D. Y. Kim, W. Luo, R. Ahuja, Y. Meng, S. Sinogeikin, J. Shu, and H.-K. Mao, Proc. Natl. Acad. Sci. USA 107, 9965 (2010).

${ }^{14}$ I. Errea, B. Rousseau, and A. Bergara, Phys. Rev. Lett. 106, 165501 (2011).

${ }^{15}$ R. A. Stager and H. G. Drickamer, Phys. Rev. 131, 2524 (1963).

${ }^{16}$ T. Yabuuchi, T. Matsuoka, Y. Nakamoto, and K. Shimizu, J. Phys. Soc. Jpn. 75, 083703 (2006).

${ }^{17}$ M. Sakata, Y. Nakamoto, K. Shimizu, T. Matsuoka, and Y. Ohishi, Phys. Rev. B 83, 220512 (2011).

${ }^{18}$ T. Matsuoka and K. Shimizu, Nature (London) 458, 186 (2009).

${ }^{19}$ M. Marqués, M. I. McMahon, E. Gregoryanz, M. Hanfland, C. L. Guillaume, C. J. Pickard, G. J. Ackland, and R. J. Nelmes, Phys. Rev. Lett. 106, 095502 (2011).

${ }^{20}$ Y. Ma, M. Eremets, A. R. Oganov, Y. Xie, I. Trojan, S. Medvedev, A. O. Lyakhov, M. Valle, and V. Prakapenka, Nature (London) 458, 182 (2009).

${ }^{21}$ E. Gregoryanz, L. F. Lundegaard, M. I. McMahon, C. Guillaume, R. J. Nelmes, and M. Mezouar, Science 320, 1054 (2008).

${ }^{22}$ A. Lazicki, A. F. Goncharov, V. V. Struzhkin, R. E. Cohen, Z. Liu, E. Gregoryanz, C. Guillaume, H.-K. Mao, and R. J. Hemley, Proc. Natl. Acad. Sci. USA 106, 6525 (2009).

${ }^{23}$ V. M. Silkin, A. Rodriguez-Prieto, A. Bergara, E. V. Chulkov, and P. M. Echenique, Phys. Rev. B 75, 172102 (2007).

${ }^{24}$ A. Rodriguez-Prieto, V. M. Silkin, A. Bergara, and P. M. Echenique, New J. Phys. 10, 053035 (2008).

${ }^{25}$ I. Errea, A. Rodriguez-Prieto, B. Rousseau, V. M. Silkin, and A. Bergara, Phys. Rev. B 81, 205105 (2010).

${ }^{26}$ H. Ehrenreich and H. R. Philipp, Phys. Rev. 128, 1622 (1962).

${ }^{27}$ M. A. Cazalilla, J. S. Dolado, A. Rubio, and P. M. Echenique, Phys. Rev. B 61, 8033 (2000).

${ }^{28}$ E. A. Taft and H. R. Philipp, Phys. Rev. 138, A197 (1965).

${ }^{29}$ T. Pichler, M. Knupfer, M. S. Golden, J. Fink, A. Rinzler, and R. E. Smalley, Phys. Rev. Lett. 80, 4729 (1998).
${ }^{30}$ V. P. Zhukov, V. M. Silkin, E. V. Chulkov, and P. M. Echenique, Phys. Rev. B 64, 180507 (2001).

${ }^{31}$ Y. Q. Cai, P. C. Chow, O. D. Restrepo, Y. Takano, K. Togano, H. Kito, H. Ishii, C. C. Chen, K. S. Liang, C. T. Chen, S. Tsuda, S. Shin, C. C. Kao, W. Ku, and A. G. Eguiluz, Phys. Rev. Lett. 97, 176402 (2006).

${ }^{32}$ L. A. Grunes and J. J. Ritsko, Phys. Rev. B 28, 3439 (1983).

${ }^{33}$ H. Abe, M. Terauchi, M. Tanaka, and S. Shin, Jpn. J. Appl. Phys. 37, 584 (1998).

${ }^{34}$ A. Rodriguez-Prieto, A. Bergara, V. M. Silkin, and P. M. Echenique, Phys. Rev. B 74, 172104 (2006).

${ }^{35}$ A. Rodriguez-Prieto and A. Bergara, Phys. Rev. B 72, 125406 (2005).

${ }^{36}$ I. G. Gurtubay, B. Rousseau, and A. Bergara, Phys. Rev. B 82, 085113 (2010)

${ }^{37}$ N. Marzari and D. Vanderbilt, Phys. Rev. B 56, 12847 (1997).

${ }^{38}$ I. Souza, N. Marzari, and D. Vanderbilt, Phys. Rev. B 65, 035109 (2001).

${ }^{39}$ P. Hohenberg and W. Kohn, Phys. Rev. 136, B864 (1964).

${ }^{40}$ E. Runge and E. K. U. Gross, Phys. Rev. Lett. 52, 997 (1984).

${ }^{41}$ M. Petersilka, U. J. Gossmann, and E. K. U. Gross, Phys. Rev. Lett. 76, 1212 (1996).

${ }^{42}$ G. Onida, L. Reining, and A. Rubio, Rev. Mod. Phys. 74, 601 (2002).

${ }^{43}$ S. L. Adler, Phys. Rev. 126, 413 (1962).

${ }^{44}$ D. Pines and P. Nozières, The Theory of Quantum Liquids, Vol. 1 (Benjamin, New York, 1966).

${ }^{45}$ W. M. Saslow and G. F. Reiter, Phys. Rev. B 7, 2995 (1973).

${ }^{46}$ J. R. Yates, X. Wang, D. Vanderbilt, and I. Souza, Phys. Rev. B 75, 195121 (2007).

${ }^{47}$ B. Rousseau, A. Eiguren, and A. Bergara, Phys. Rev. B 85, 054305 (2012).

${ }^{48}$ P. Giannozzi et al., J. Phys.: Condens. Matter 21, 395502 (2009).

${ }^{49}$ A. A. Mostofi, J. R. Yates, Y.-S. Lee, I. Souza, D. Vanderbilt, and N. Marzari, Comput. Phys. Commun. 178, 685 (2008).

${ }^{50}$ J. P. Perdew, K. Burke, and M. Ernzerhof, Phys. Rev. Lett. 77, 3865 (1996).

${ }^{51}$ P. O. Nilsson and G. Forssell, Phys. Rev. B 16, 3352 (1977).

${ }^{52}$ L. B. Leder, Phys. Rev. 103, 1721 (1956).

${ }^{53}$ C. J. Powell, Proc. Phys. Soc. 76, 593 (1960).

${ }^{54}$ J. L. Robins and P. E. Best, Proc. Phys. Soc. 79, 110 (1962).

${ }^{55}$ B. R. Cooper, H. Ehrenreich, and H. R. Philipp, Phys. Rev. 138, A494 (1965).

${ }^{56}$ A. D. Becke and K. E. Edgecombe, J. Chem. Phys. 92, 5397 (1990).

${ }^{57}$ S. Lei, D. A. Papaconstantopoulos, and M. J. Mehl, Phys. Rev. B 75, 024512 (2007).

${ }^{58}$ I. Loa, K. Syassen, G. Monaco, G. Vankó, M. Krisch, and M. Hanfland, Phys. Rev. Lett. 107, 086402 (2011).

${ }^{59}$ H.-K. Mao, Y. Ding, Y. Xiao, P. Chow, J. Shu, S. Lebègue, A. Lazicki, and R. Ahuja, Proc. Natl. Acad. Sci. USA 108, 20434 (2011). 